\title{
ODD PERFECT NUMBERS HAVE A PRIME FACTOR EXCEEDING $10^{8}$
}

\author{
TAKESHI GOTO AND YASUO OHNO
}

\begin{abstract}
Jenkins in 2003 showed that every odd perfect number is divisible by a prime exceeding $10^{7}$. Using the properties of cyclotomic polynomials, we improve this result to show that every perfect number is divisible by a prime exceeding $10^{8}$.
\end{abstract}

\section{INTRODUCTION}

A positive integer $n$ is said to be perfect if $\sigma(n)=2 n$, where $\sigma(n)$ denotes the sum of positive divisors of $n$. As of November 2006, forty-four even perfect numbers are known. On the other hand, it is still open whether or not an odd perfect number exists. Many necessary conditions for existence of an odd perfect number have been found. For example, Euler showed that the prime factorization of an odd perfect number $n$ must be of the form

$$
n=p_{0}^{e_{0}} p_{1}^{2 e_{1}} \cdots p_{k}^{2 e_{k}}, \quad p_{0} \equiv e_{0} \equiv 1 \quad(\bmod 4) .
$$

Here $p_{0}$ is called the special prime of $n$. Brent, Cohen and te Riele [1] showed that $n>10^{300}$. Chein [2] and Hagis [7] independently showed that $n$ must have at least 8 distinct prime factors, and this bound was recently improved to 9 by Nielsen [18]. Hare 9] showed that $n$ must have totally at least 47 prime factors, and he recently improved this bound to 75 in [10.

In the present paper, we focus our attention on the largest prime factor of an odd perfect number. In 1944, Kanold [15] showed that every odd perfect number is divisible by a prime exceeding 60 . This lower bound was improved by Hagis and McDaniel [5 (resp. 6]) to $10^{4}$ (resp. $10^{5}$ ), by Hagis and Cohen 8 to $10^{6}$, by Jenkins [13, 14] to $10^{7}$. Jenkins reported that he needed about 25,800 hours for computing time. On the other hand, Ore [19] proved that every perfect number is a harmonic number (a positive integer is said to be harmonic if the harmonic mean of its positive divisors is an integer). Chishiki, Goto and Ohno 3 showed that every odd harmonic number is divisible by a prime exceeding $10^{5}$. This is another extension of the result given by McDaniel [6]. The aim of the present paper is to show the following result.

Theorem 1.1. Every odd perfect number is divisible by a prime exceeding $10^{8}$.

Received by the editor December 13, 2006 and, in revised form, February 26, 2007.

2000 Mathematics Subject Classification. Primary 11A25, 11 Y70.

Key words and phrases. Odd perfect numbers, cyclotomic numbers.

This work was supported by Computing and Communications Center, Kyushu University.

The second author was supported in part by JSPS Grant-in-Aid No. 15740025 and No. 18740020 .

(C)2008 American Mathematical Society Reverts to public domain 28 years from publication 
In $\S 2$ and $\S 3$, we explain our proof of Theorem 1.1. For more details, see the note on the webpage http://www.ma.noda.tus.ac.jp/u/tg/perfect.html. The programs used in the computation are also available on this webpage. In $\S 4$, we discuss our algorithm.

\section{Outline of THE PROOF}

In this paper, $p, q$ will denote odd primes and $r$ will denote a (possibly even) prime. The $d$ th cyclotomic polynomial will be denoted by $\Phi_{d}$, so that $\sigma\left(p^{e}\right)=$ $1+p+p^{2}+\cdots+p^{e}=\prod_{d \mid(e+1), d>1} \Phi_{d}(p)$. Let $n$ be an odd perfect number whose prime factorization is $p_{1}^{e_{1}} \cdots p_{k}^{e_{k}}$ (note that this is different from the form in $\S 1$ ). Since $\sigma$ is multiplicative, it easily follows from $\sigma(n)=2 n$ that

$$
\prod_{i=1}^{k} \prod_{\substack{d \mid\left(e_{i}+1\right) \\ d>1}} \Phi_{d}\left(p_{i}\right)=2 \prod_{i=1}^{k} p_{i}^{e_{i}}
$$

For integers $a, d \geq 2$, the integer $\Phi_{d}(a)$ is often called a cyclotomic number. In the nineteenth century, cyclotomic numbers were studied by Sylvester, Kronecker et al. The following proposition is a summary of their results (cf. 20]).

Proposition 2.1. Let $q$ be a prime, and $a, d$ be integers. Suppose that $a \geq 2, d \geq 3$. Then the following facts hold.

(1) If $q \mid \Phi_{d}(a)$, then $q \mid d$ or $q \equiv 1(\bmod d)$.

(2) If $q \mid \Phi_{d}(a)$ and $q \mid d$, then $q^{2} \nmid \Phi_{d}(a)$.

(3) If $(a, d) \neq(2,6)$, then the cyclotomic number $\Phi_{d}(a)$ has at least one prime factor $q$ such that $q \equiv 1(\bmod d)$.

2.1. Acceptable values. Assume that $n$ is an odd perfect number whose largest prime factor is less than $10^{8}$. Then the right-hand side of (2.1) has no prime factors exceeding $10^{8}$, hence so does the left-hand side. Since $n$ is odd, the cyclotomic numbers in (2.1) are not divisible by 4 .

Definition. For an odd prime $p$ and a prime $r$, we say that $\Phi_{r}(p)$ is acceptable if the following two conditions hold.

(1) $\Phi_{r}(p)$ has no prime factors exceeding $10^{8}$.

(2) $4 \nmid \Phi_{r}(p)$.

Clearly, cyclotomic numbers in (2.1) must be acceptable. Our first aim is to find all acceptable values $\Phi_{r}(p)$ with $3 \leq p<10^{8}$.

Lemma 2.2. Suppose that $3 \leq p<10^{8}, r \geq 7$, and the cyclotomic number $\Phi_{r}(p)$ is acceptable. Then $\Phi_{r}(p)$ is one of 671 numbers listed in the online note mentioned in $\S 1$.

The details of the proof of Lemma 2.2 can be found in $\S 3$. The basic techniques used for proving Lemma 2.2 is first to show that we can restrict our search to $r<5 \times 10^{7}$ (hence we need search only finitely many numbers), and then to use properties of cyclotomic polynomials to further refine the search. 
2.2. Inadmissible small primes. Under Lemma 2.2, we show the following.

Lemma 2.3. Assume that $n$ is an odd perfect number whose largest prime factor is less than $10^{8}$. Then $n$ is not divisible by any prime in the following set $X$.

$$
\begin{aligned}
& X=\{3,5,7,11,13,17,19,23,29,31,37,43,61,71,113,127,131,151,197, \\
& 211,239,281,337,379,421,449,463,491,547,617,631,659,673,743, \\
&757,827,911,953,967,1051,1093\} .
\end{aligned}
$$

In particular, $n$ has no prime factors less than 41.

For the proof of this lemma, primes in $X$ are considered in the order

$$
\begin{aligned}
& 1093,151,31,127,19,11,7,23,131,37,61,13,3,5,29,43,1051,17, \\
& 71,113,197,211,239,281,337,379,421,449,463,491,547,617,631, \\
& 659,673,743,757,827,911,953,967 .
\end{aligned}
$$

For example, after proving that $1093 \nmid n$, we prove $151 \nmid n$ as follows. Assume that $151 \mid n$. Then the left-hand side of (2.1) is divisible by a cyclotomic number $\Phi_{r}(151)$ for some prime $r$. Only $\Phi_{3}(151)=3 \cdot 7 \cdot 1093$ is acceptable, however, it is contradictory to $1093 \nmid n$. Hence we have $151 \nmid n$. The proof of $1093 \nmid n$ has many branches. At each step, we only need to branch on $r$ where $\Phi_{r}(p)$ is acceptable. We write $p^{*}$ to indicate that $p$ is the special prime. Note that two different primes cannot be special simultaneously.

(initial part of the proof of Lemma 2.3.

$(1093 \nmid n)$

$1093: \Phi_{2}(1093)=2 \cdot 547 ; \Phi_{3}(1093)=3 \cdot 398581$.

$1093^{*}, 547: \Phi_{3}(547)=3 \cdot 163 \cdot 613$.

$1093^{*}, 547,613: \Phi_{3}(613)=3 \cdot 7 \cdot 17923 ; \Phi_{5}(613)=131 \cdot 20161 \cdot 53551$.

$1093^{*}, 547,613,17923: \Phi_{3}(17923)=3 \cdot 13 \cdot 31 \cdot 265717$.

$1093^{*}, 547,613,17923,265717$ : Any $\Phi_{r}(265717)$ is unacceptable.

$1093^{*}, 547,613,20161$ : Any $\Phi_{r}(20161)$ is unacceptable.

1093, 398581: $\Phi_{2}(398581)=2 \cdot 17 \cdot 19 \cdot 617 ; \Phi_{3}(398581)=3 \cdot 1621 \cdot 32668561$.

$1093,398581^{*}, 617: \Phi_{3}(617)=97 \cdot 3931$.

$1093,398581^{*}, 617,3931: \Phi_{3}(3931)=3 \cdot 7 \cdot 31 \cdot 23743$.

$1093,398581^{*}, 617,3931,23743: \Phi_{3}(23743)=3 \cdot 37 \cdot 5078863$.

$1093,398581^{*}, 617,3931,23743,5078863$ : Any $\Phi_{r}(5078863)$ is unacceptable.

1093, 398581, 32668561: $\Phi_{2}(32668561)=2 \cdot 19 \cdot 43 \cdot 19993$.

$1093,398581,32668561^{*}, 19993: \Phi_{3}(19993)=3 \cdot 73 \cdot 1825297$.

$1093,398581,32668561^{*}, 19993,1825297: \Phi_{3}(1825297)=3 \cdot 326863 \cdot 3397663$.

$1093,398581,32668561^{*}, 19993,1825297,326863: \Phi_{3}(326863)=3 \cdot 67 \cdot 3313 \cdot 160441$.

$1093,398581,32668561^{*}, 19993,1825297,326863,3313: \Phi_{3}(3313)=3 \cdot 7 \cdot 7 \cdot 19 \cdot 3931$.

1093, 398581, 32668561* 19993, 1825297, 326863, 3313, 3931 :

$\Phi_{3}(3931)=3 \cdot 7 \cdot 31 \cdot 23743$.

1093,398581,32668561* $19993,1825297,326863,3313,3931,23743$ :

$\Phi_{3}(23743)=3 \cdot 37 \cdot 5078863$.

1093, 398581, 32668561* ${ }^{*}, 19993,1825297,326863,3313,3931,23743,5078863$ :

Any $\Phi_{r}(5078863)$ is unacceptable.

$(153 \nmid n)$

$151: \Phi_{3}(151)=3 \cdot 7 \cdot 1093$.

151,1093 : contradiction to $1093 \nmid n$.

$(31 \nmid n)$

$31: \Phi_{3}(31)=3 \cdot 331 ; \Phi_{5}(31)=5 \cdot 11 \cdot 17351 ; \Phi_{13}(31)=42407 \cdot 2426789 \cdot 7908811$. $\ldots$ 
For the complete proof of Lemma 2.3, see the online note mentioned in $\S 1$.

2.3. Restriction on exponents in the prime factorization. Under Lemmas $2.2,2.3$, we show the following.

Lemma 2.4. Assume that $n$ is an odd perfect number whose largest prime factor is less than $10^{8}$. Let the prime factorization of $n$ be $p_{1}^{e_{1}} \cdots p_{k}^{e_{k}}$. Then every $e_{i}+1$ has no prime factors greater than 5 .

Assume that some $e_{i}+1$ has a prime factor $r$ greater than 5 . Then the cyclotomic number $\Phi_{r}\left(p_{i}\right)$ is acceptable. Hence $\Phi_{r}\left(p_{i}\right)$ is one of the 671 numbers mentioned in the statement of Lemma 2.2. From Lemma 2.3. it follows that $p_{i} \notin X$, and $\Phi_{r}\left(p_{i}\right)$ is not divisible by any prime in $X$. There are 87 such cyclotomic numbers. It is sufficient to show that each of these 87 numbers cannot appear in (2.1). For example, we can check $\Phi_{13}(47)$ as follows. Assume that $\Phi_{13}(47) \mid n$. This implies $14050609 \mid n$ since

$$
\Phi_{13}(47)=53 \cdot 2237 \cdot 14050609 \cdot 71265169 .
$$

Only $\Phi_{2}(14050609)=2 \cdot 5 \cdot 7 \cdot 200723$ is acceptable. Since $5 \in X$, this is contradictory to Lemma 2.3. For the complete proof of Lemma 2.4, see the online note mentioned in $\S 1$.

2.4. Four sets. We denote by $|A|$ the size of the set $A$. Let $P=\{p \mid p$ is prime, $\left.41 \leq p<10^{8}\right\}$. A computer search showed that $|P|=5761443$ and

$$
P^{*}:=\prod_{p \in P} \frac{p}{p-1}<4.87934286481804236682 .
$$

The four subsets $S, T, U, V$ of $P$ are defined by

$$
\begin{aligned}
& S=\{p \in P \mid p \not \equiv 1(\bmod 3) \text { and } p \not \equiv 1(\bmod 5)\}, \\
& T=\{p \in P \mid p \equiv 1(\bmod 15)\}, \\
& U=\left\{p \in P \mid p \equiv 1(\bmod 3), p \not \equiv 1(\bmod 5) \text { and } \Phi_{5}(p) \text { is unacceptable }\right\}, \\
& V=\left\{p \in P \mid p \not \equiv 1(\bmod 3), p \equiv 1(\bmod 5) \text { and } \Phi_{3}(p) \text { is unacceptable }\right\} .
\end{aligned}
$$

Note that these subsets are disjoint and $S \cup T \cup U \cup T \neq P$. Computer searches showed that $|S|=2160618,|T|=719983,|U|=2144188,|V|=496701$ and

$$
\begin{aligned}
S^{*} & :=\prod_{p \in S} \frac{p}{p-1}>1.82219345901032950583, \\
T^{*} & :=\prod_{p \in T} \frac{p}{p-1}>1.19902263543776496408, \\
U^{*} & :=\prod_{p \in U} \frac{p}{p-1}>1.43699138263382743310, \\
V^{*} & :=\prod_{p \in V} \frac{p}{p-1}>1.03750936160818766647 .
\end{aligned}
$$

Proposition 2.5. Assume that $n$ is an odd perfect number whose largest prime factor is less than $10^{8}$. Then the following facts hold.

(1) The number $n$ is divisible by at most two elements of $S$. If there is such an element $s$, then it is not the special prime of $n$, and $s \geq 47$. 
(2) The number $n$ is divisible by at most one element of $T$. If there is such an element $t$, then it is the special prime of $n$, and $t \geq 61$.

(3) The number $n$ is divisible by at most one element of $U$. If there is such an element $u$, then it is the special prime of $n$, and $u \geq 73$.

(4) The number $n$ is not divisible by any element of $V$.

Proof. These facts can be proven similarly to the paper 8 . Here we show only (1) since it is slightly different from the original one. Suppose that $p \in S$ and $p \mid n$. Then $p$ divides some cyclotomic number $\Phi_{d}\left(p_{j}\right)$ in equation (2.1). Assume that $d \neq 2$. By Lemma 2.4 $d$ is divisible by 3 or 5 , and hence $p \equiv 1(\bmod 3)$ or $p \equiv 1$ $(\bmod 5)$ from Proposition 2.1(1). This is a contradiction to $p \in S$. Therefore $d=2$. Because of the definition of the special prime, $p_{j}$ is the special prime and $p$ is not. Since the smallest element of $S$ is 47 , it holds that $p \geq 47$. Since $p \mid \Phi_{2}\left(p_{j}\right)$ and the other cyclotomic numbers in (2.1) are not divisible by $p$, we have $p^{2} \mid\left(p_{j}+1\right)$. If there are three such $p$, then $p_{j}+1 \geq 2 \cdot 47^{2} \cdot 53^{2} \cdot 59^{2}>10^{8}$, a contradiction to $p_{j}<10^{8}$.

We define $\sigma_{-1}(n)$ by

$$
\sigma_{-1}(n):=\sum_{d \mid n} d^{-1}=\frac{\sigma(n)}{n} .
$$

An integer $n$ is perfect if and only if $\sigma_{-1}(n)=2$. The function $\sigma_{-1}$ is multiplicative, and for any positive integer $e$,

$$
\sigma_{-1}\left(p^{e}\right)<\sigma_{-1}\left(p^{\infty}\right):=\lim _{e \rightarrow \infty} \sigma_{-1}\left(p^{e}\right)=\frac{p}{p-1} .
$$

Assume again that $n=p_{1}^{e_{1}} \cdots p_{k}^{e_{k}}$ is an odd perfect number whose largest prime factor is less than $10^{8}$. From Proposition 2.5 $n$ is divisible by at most three elements of $S \cup T \cup U \cup V$. Since $x /(x-1)$ is monotone decreasing for $x>1$, if $p_{i} \in S$, then $\sigma_{-1}\left(p_{i}^{\infty}\right) \leq 47 / 46$, and if $p_{i} \in T \cup U$, then $\sigma_{-1}\left(p_{i}^{\infty}\right) \leq 61 / 60$. Therefore it follows from Proposition 2.5 that

$$
2=\sigma_{-1}(n)<\prod_{i} \frac{p_{i}}{p_{i}-1} \leq \frac{47}{46} \cdot \frac{53}{52} \cdot \frac{61}{60} \cdot \frac{P^{*}}{S^{*} T^{*} U^{*} V^{*}}<1.5859314817,
$$

a contradiction. The proof of Theorem 1.1 is completed.

\section{Details on the Search For acCeptable values}

From Proposition 2.1 (3), the cyclotomic number $\Phi_{r}(p)$ has a prime factor $q$ such that $q \geq 2 r+1$, hence if $\Phi_{r}(p)$ is acceptable, then $r<5 \cdot 10^{7}$. Therefore we need to check only finitely many cyclotomic numbers, however, it is hard to directly determine whether or not each number is acceptable because of difficulty of prime factorization. The key point of the proof of Lemma 2.2 is the following lemma. The notation $p^{e} \| n$ means that $p^{e} \mid n$ and $p^{e+1} \nmid n$.

Lemma 3.1. If $p, q<10^{8}$ and $6679<r<5 \cdot 10^{7}$, then $q^{4} \nmid \Phi_{r}(p)$ for all primes $q$ and $q^{3} \| \Phi_{r}(p)$ for at most one prime $q$. In fact, $q^{3} \nmid \Phi_{r}(p)$ except for

$28499^{3}\left\|\Phi_{14249}(70081199), 60647^{3}\right\| \Phi_{30323}(6392117), 63587^{3} \| \Phi_{31793}(42326917)$.

In the rest of this section, we prove Lemma 2.2 using Lemma 3.1. In $\S 4$, we discuss our algorithm to show Lemma 3.1 . 
Lemma 3.2. If $10^{2}<p<10^{8}$ and $6679<r<5 \cdot 10^{7}$, then $\Phi_{r}(p)$ is unacceptable.

Proof. For a prime $r$, we define $Q(r)$ by

$$
Q(r)=\prod_{\substack{q<10^{8} \\ q \equiv 1(\bmod r)}} q .
$$

Note that $r \cdot Q(r)$ is the product of all primes which may divide an acceptable cyclotomic number $\Phi_{r}(p)$ in view of Proposition 2.1. Therefore, if $\Phi_{r}(p)$ is acceptable and squarefree, then $\Phi_{r}(p) \leq r \cdot Q(r)$. Under the assumption of this lemma, we have $\Phi_{r}(p)<10^{8} r \cdot Q(r)^{2}$ from Lemma 3.1.

We show that if $6679<r<5 \cdot 10^{7}$, then $10^{8} r \cdot Q(r)^{2}<10^{2(r-1)}$. A direct computation showed that if $6679<r<5 \cdot 10^{4}$, then the required inequality holds. Suppose that $r \geq 5 \cdot 10^{4}$. If $q \equiv 1(\bmod r)$, then $q=2 k r+1$ with $k<10^{3}$, hence we have $10^{4} \sqrt{r} \cdot Q(r)<\left(10^{8}\right)^{10^{3}+2}<10^{10^{4}}<10^{r-1}$. The square of this inequality is the required one.

Hence it follows that $\Phi_{r}(p)<10^{8} r \cdot Q(r)^{2}<10^{2(r-1)}$. On the other hand, we have $\Phi_{r}(p)>p^{r-1}>10^{2(r-1)}$, a contradiction.

Lemma 3.3. If $10^{2}<p<10^{8}$ and $4723<r \leq 6679$, then $\Phi_{r}(p)$ is unacceptable.

Proof. A direct computation showed that if $4723<r \leq 6679$, then $10^{8} r \cdot Q(r)<$ $10^{2(r-1)}$. Another computer search showed that if $4723<r \leq 6679$, then $q^{3} \nmid$ $\Phi_{r}(p)$ for all primes $q$ and $q^{2} \| \Phi_{r}(p)$ for at most one prime $q$. Assume that $10^{2}<p<10^{8}, 4723<r \leq 6679$ and $\Phi_{r}(p)$ is acceptable. Then it follows that $10^{2(r-1)}<p^{r-1}<\Phi_{r}(p)<10^{8} r \cdot Q(r)<10^{2(r-1)}$, a contradiction.

Lemma 3.4. (1) If $10^{6}<p<10^{8}$ and $2707<r \leq 4723$, then $\Phi_{r}(p)$ is unacceptable. (2) If $10^{7}<p<10^{8}$ and $2503<r \leq 2707$, then $\Phi_{r}(p)$ is unacceptable.

Proof. A computer search showed that if $p, q<10^{8}$ and $2503<r \leq 4723$, then $q^{2} \mid \Phi_{r}(p)$ for at most one prime $q$, and $q^{3} \nmid \Phi_{r}(p)$ except for

$$
\begin{array}{lll}
10709^{3} \| \Phi_{2677}(6619441), & 5939^{3} \| \Phi_{2969}(41492783), & 6719^{3} \| \Phi_{3359}(59698039), \\
8147^{3} \| \Phi_{4073}(41112823), & 8147^{3} \| \Phi_{4073}(41728717) . &
\end{array}
$$

(1) A direct computation showed that if $2707<r \leq 4723$, then $\left(10^{8}\right)^{2} r \cdot Q(r)<$ $10^{6(r-1)}$. Hence if $10^{6}<p<10^{8}, 2707<r \leq 4723$ and $\Phi_{r}(p)$ is acceptable, then it follows that $10^{6(r-1)}<p^{r-1}<\Phi_{r}(p)<\left(10^{8}\right)^{2} r \cdot Q(r)<10^{6(r-1)}$, a contradiction.

(2) A direct computation showed that if $2503<r \leq 2707$, then $\left(10^{8}\right)^{2} r \cdot Q(r)<$ $10^{7(r-1)}$. Hence if $10^{7}<p<10^{8}, 2503<r \leq 2707$ and $\Phi_{r}(p)$ is acceptable, then it follows that $10^{7(r-1)}<p^{r-1}<\Phi_{r}(p)<\left(10^{8}\right)^{2} r \cdot Q(r)<10^{7(r-1)}$, a contradiction.

Suppose that $p<10^{2}$ and $q^{2} \mid \Phi_{r}(p)$. Proposition 2.1 (1), (2) imply that $r \mid(q-1)$, and we have $p^{r} \equiv 1\left(\bmod q^{2}\right)$ by the argument in the proof of Proposition 4.1. Hence it follows that $p^{q-1} \equiv 1\left(\bmod q^{2}\right)$. According to the table of Montgomery [16], all solutions of $p^{q-1} \equiv 1\left(\bmod q^{2}\right), 3 \leq p<10^{2}, q<10^{8}$ are given by Table 1 . From the table, if $p<10^{2}$ and $3 \leq r<5 \cdot 10^{7}$, then $\Phi_{r}(p)$ is squarefree except for

$$
\begin{aligned}
& 11^{2}\left\|\Phi_{5}(3), 48947^{2}\right\| \Phi_{24473}(17), 47^{2}\left\|\Phi_{23}(53), 59^{2}\right\| \Phi_{29}(53), \\
& 7^{2}\left\|\Phi_{3}(67), 47^{2}\right\| \Phi_{23}(71), 7^{2}\left\|\Phi_{3}(79), 4871^{2}\right\| \Phi_{487}(83) .
\end{aligned}
$$


TABLE 1

\begin{tabular}{rl|ll}
\hline \multicolumn{1}{c|}{$q$} & $p$ & \multicolumn{1}{c}{$q$} \\
\hline 3 & 11,1006003 & 43 & 5,103 \\
5 & $20771,40487,53471161$ & 47 & none \\
7 & 5,491531 & 53 & $3,47,59,97$ \\
11 & 71 & 59 & 2777 \\
13 & 863,1747591 & 61 & none \\
17 & $3,46021,48947$ & 67 & $7,47,268573$ \\
19 & $3,7,13,43,137,63061489$ & 71 & $3,47,331$ \\
23 & $13,2481757,13703077$ & 73 & 3 \\
29 & none & 79 & $7,263,3037,1012573,60312841$ \\
31 & $7,79,6451,2806861$ & 83 & 4871,13691 \\
37 & 3,77867 & 89 & 3,13 \\
41 & 29,1025273 & 97 & 7,2914393 \\
\hline
\end{tabular}

Using a program based on Algorithm 2 in $\S 4$, we showed this fact again without Montgomery's table.

For a prime $p$, we define $R(p)$ by

$$
R(p)=\min \left\{a \in \mathbb{N} \mid 10^{8} r \cdot Q(r)<p^{r-1} \text { if } r \geq a\right\} .
$$

It immediately follows that $R(p) \leq 5 \cdot 10^{4}$. In fact, if $r \geq 5 \cdot 10^{4}$, then

$$
10^{8} r \cdot Q(r)<\left(10^{8}\right)^{10^{3}+2}<\left(3^{17}\right)^{10^{3}+2}<3^{r-1} \leq p^{r-1} .
$$

By directly checking each $r<5 \cdot 10^{4}$, we have Table 2 .

TABLE 2

\begin{tabular}{rc|cc|cc}
\hline$p$ & $R(p)$ & $p$ & $R(p)$ & $p$ & $R(p)$ \\
\hline 3 & 9650 & 29 & 5508 & 61 & 4952 \\
5 & 7950 & 31 & 5508 & 67 & 4890 \\
7 & 7238 & 37 & 5310 & 71 & 4878 \\
11 & 6548 & 41 & 5262 & 73 & 4878 \\
13 & 6318 & 43 & 5262 & 79 & 4818 \\
17 & 5954 & 47 & 5108 & 83 & 4788 \\
19 & 5882 & 53 & 5060 & 89 & 4734 \\
23 & 5660 & 59 & 4988 & 97 & 4724 \\
\hline
\end{tabular}

Lemma 3.5. If $3 \leq p<10^{2}$ and $R(p) \leq r<5 \cdot 10^{7}$, then $\Phi_{r}(p)$ is unacceptable.

Proof. Assume that $\Phi_{r}(p)$ is acceptable. Since $q^{2} \mid \Phi_{r}(p)$ for at most one prime $q$, it follows that $p^{r-1}<\Phi_{r}(p)<10^{8} r \cdot Q(r)<p^{r-1}$, a contradiction.

For a completion of the proof of Lemma 2.2, we must check $r$ given in Table 3 for each $p$. We can do this by a direct search.

TABle 3

\begin{tabular}{cc}
\hline$p$ & $r$ \\
\hline $3 \leq p<10^{2}$ & $r \leq R(p)-1$ \\
$10^{2}<p<10^{6}$ & $r \leq 4723$ \\
$10^{6}<p<10^{7}$ & $r \leq 2707$ \\
$10^{7}<p<10^{8}$ & $r \leq 2503$ \\
\hline
\end{tabular}




\section{IMPROVED ALGORITHM}

It is the hardest task to show Lemma 3.1. In general, we consider the algorithm to find all triplets of odd primes $(p, q, r)$ satisfying $p, q<M, r \geq c$ and $q^{3} \mid \Phi_{r}(p)$ for some given integers $M, c$. From Proposition 2.1, it is necessary that $r<M / 2$ and $q \equiv 1(\bmod 2 r)$. The algorithm made by Jenkins $[13]$ is described by Algorithm 1 .

\section{Algorithm 1 (original algorithm)}

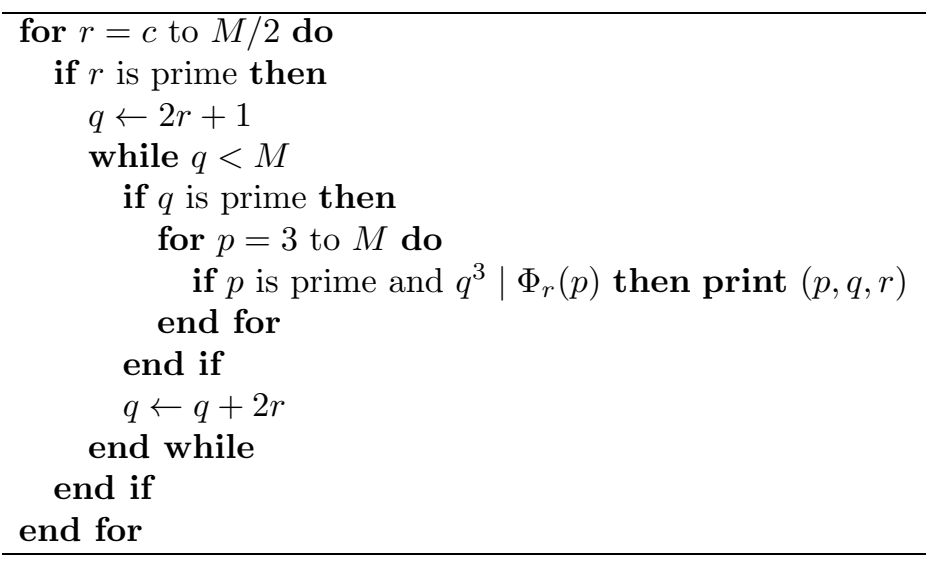

By this algorithm, we will check triplets $(p, q, r)$ in the set

$$
\{(p, q, r) \mid p, q, r \text { are primes, } p, q<M, c \leq r<M / 2, q \equiv 1(\bmod 2 r)\} .
$$

Our improved algorithm is based on the following proposition.

Proposition 4.1. Suppose that $q \mid \Phi_{r}(p)$ and $q \equiv 1(\bmod r)$. Let $g$ be a generator of the cyclic group $\left(\mathbb{Z} / q^{m} \mathbb{Z}\right)^{\times}$, and put $w=(q-1) / r$. Then $q^{m} \mid \Phi_{r}(p)$ if and only if $p$ belongs to the subgroup $\left\langle g^{w q^{m-1}}\right\rangle$ of $\left(\mathbb{Z} / q^{m} \mathbb{Z}\right)^{\times}$.

Proof. Note that $w$ is an integer since $q \equiv 1(\bmod r)$. Assume that $p \equiv 1(\bmod q)$. Then it follows that $\Phi_{r}(p) \equiv r(\bmod q)$, a contradiction. Hence $p \not \equiv 1(\bmod q)$ and

$$
\begin{aligned}
q^{m} \mid \Phi_{r}(p) & \Longleftrightarrow q^{m} \mid(p-1) \Phi_{r}(p) \\
& \Longleftrightarrow p^{r} \equiv 1 \quad\left(\bmod q^{m}\right) \\
& \Longleftrightarrow \text { the order of } p \in\left(\mathbb{Z} / q^{m} \mathbb{Z}\right)^{\times} \text {is } r \\
& \Longleftrightarrow p \in\left\langle g^{w q^{m-1}}\right\rangle,
\end{aligned}
$$

as required.

Our program is described by Algorithm 2. In the algorithm, we use Proposition 4.1 with $m=2$ instead of $m=3$, since we would not like to deal with large numbers.

By the improved algorithm, we will check triplets $(p, q, r)$ in the set

$$
\left\{(p, q, r) \mid p, q, r \text { are primes, } p, q<M, c \leq r<M / 2, q \equiv 1(\bmod 2 r), p \in\left\langle g^{q(q-1) / r}\right\rangle\right\},
$$

which is a subset of the set given by (4.2). Note that if $c>\sqrt{M} / 2$, then $q \geq$ $2 r+1>\sqrt{M}$ and hence $q^{2}>M$. Therefore there exists at most one prime $p<M$ satisfying $p \equiv\left(g^{q(q-1) / r}\right)^{i}\left(\bmod q^{2}\right)$ for each $i$. In [4], we estimate the amount of the computation for the improved algorithm. In $\S 5$, we give some data for the effect of the improvement. 
Algorithm 2 (improved algorithm)

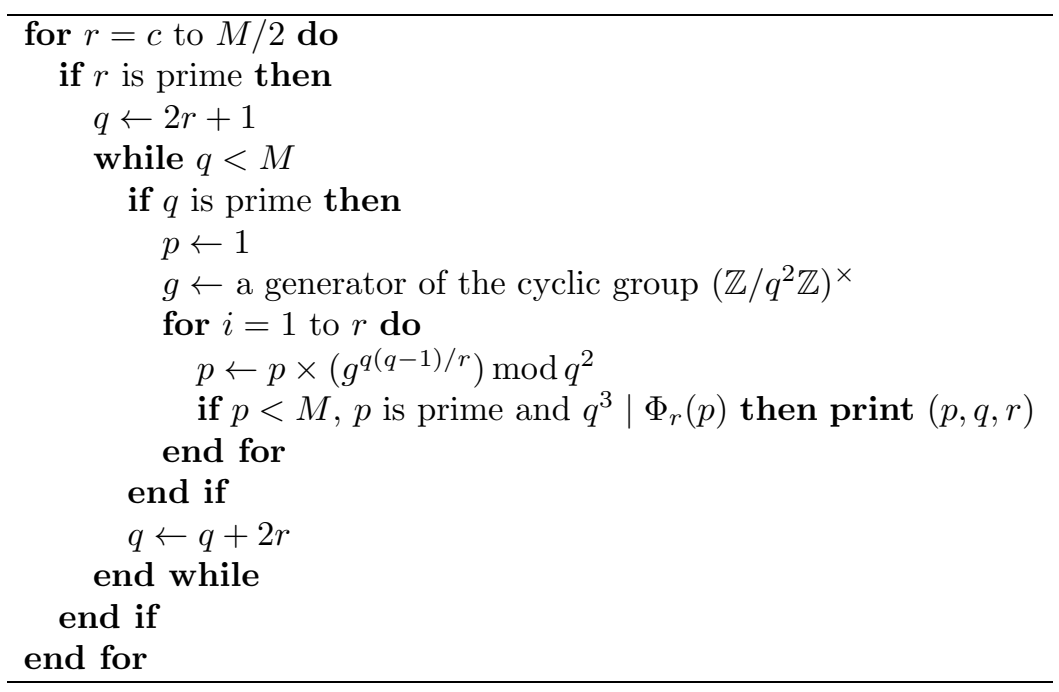

\section{Concluding Remarks}

For the proof of Lemma 3.1 we used PARI/GP and a computer AlpherServer GS320 (CPU: Alpha21264, 731MHz) which belongs to Computing and Communications Center, Kyushu University. The computation needed about 26,000 hours for total CPU time. Since we used ten CPU's simultaneously, it took about four months. For the bound $10^{7}$, the same computer needed 274 hours. Using our UBASIC program and a PC (CPU: Pentium4, 3GHz), we needed 42 hours. For the bound $10^{6}$, we needed 11 hours using the original UBASIC program made by Jenkins, and needed 35 minutes using our UBASIC program. These data show how our improved algorithm is effective.

Our UBASIC program is faster than our PARI/GP program, however, we cannot use UBASIC on UNIX machines. The authors do not have enough Windows machines, so we mainly used PARI/GP to show Lemma 3.1 and used UBASIC for the other computations.

The inequality at the end of $\S 2$ is much stronger than is needed and the theorem could be proved by only $S$ and $U$. The referee of the paper [8] also pointed out this fact. However, it is considered that the four sets are worth being mentioned as Hagis and Cohen claimed.

Iannucci [1], [12] showed that the second (resp. third) largest prime factor of an odd perfect number must exceed $10^{4}$ (resp. $10^{2}$ ). He used the bound $10^{6}$ of the largest prime factor, hence the new bound $10^{8}$ is possibly useful to raise the bounds of the second and third largest prime factor.

In order to raise the lower bound to $10^{9}$, we need much CPU time or a better method. In the case of the bound $10^{8}$, it was shown that if $\Phi_{r}(p)$ is acceptable, then $r \leq 47$. Is it possible to eliminate the possibility of $47<r<5 \cdot 10^{7}$ without hard computations? The authors consider that a hint is in the paper by Murty and Wong [17]. They showed that if the ABC conjecture is true, then largest prime factors of cyclotomic numbers are large enough in a sense. 


\section{REFERENCES}

[1] R. P. Brent, G. L. Cohen, H. J. J. te Riele, Improved techniques for lower bounds for odd perfect numbers, Math. Comp. 57 (1991), 857-868. MR.1094940 (92c:11004)

[2] J. E. Z. Chein, An Odd Perfect Number has a Least 8 Prime Factors, Ph.D. thesis, Pennsylvania State Univ., 1979.

[3] Y. Chishiki, T. Goto and Y. Ohno, On the largest prime divisor of an odd harmonic number, Math. Comp. 76 (2007), 1577-1587.

[4] T. Goto And Y. Oнno, Perfect numbers, cyclotomic numbers and ABC conjecture (Japanese), Trans. Japan Soc. Indust. Appl. Math. 16 (2006), 187-195.

[5] P. Hagis, JR. And W. L. MCDaniel, On the largest prime divisor of an odd perfect number, Math. Comp. 27 (1973), 955-957. MR0325508 (48:3855)

[6] P. Hagis, JR. And W. L. MCDaniel, On the largest prime divisor of an odd perfect number II, Math. Comp. 29 (1975), 922-924. MR0371804 (51:8021)

[7] P. Hagis, JR., Outline of a proof that every odd perfect number has at least eight prime factors, Math. Comp. 35 (1980), 1027-1032. MR.572873 (81k:10004)

[8] P. Hagis, JR. And G. L. Cohen, Every odd perfect number has a prime factor which exceeds $10^{6}$, Math. Comp. 67 (1998), 1323-1330. MR.1484897 (98k:11002)

[9] K. G. HARE, More on the total number of prime factors of an odd perfect number, Math. Comp. 74 (2005), 1003-1008. MR2114661 (2005h:11010)

[10] K. G. HARE, New techniques for bounds on the total number of prime factors of an odd perfect number, Math. Comp., to appear.

[11] D. E. IANNUCCI, The second largest prime divisor of an odd perfect number exceeds ten thousand, Math. Comp., 68 (1999), 1749-1760. MR1651761(2000i:11200)

[12] D. E. IANNUCCI, The third largest prime divisor of an odd perfect number exceeds one hundred, Math. Comp., 69 (2000), 867-879. MR1651762 (2000i:11201)

[13] P. M. Jenkins, Odd perfect numbers have a prime factor exceeding $10^{7}$, Senior Thesis, Brigham Young University, 2000.

[14] P. M. Jenkins, Odd perfect numbers have a prime factor exceeding $10^{7}$, Math. Comp. 72 (2003), 1549-1554. MR.1972752 (2004a:11002)

[15] H. J. KANOLD, Folgerungen aus dem Vorkommen einer Gauss'schen Primzahl in der Primfaktorenzerlegung einer ungeraden vollkommenen Zahl, J. Reine Angew. Math. 186 (1944), 25-29. MR0012079 (6:255c)

[16] P. L. Montgomery, New solutions of $a^{p-1} \equiv 1\left(\bmod p^{2}\right)$, Math. Comp. 61 (1993), 361-363. MR.1182246 (94d:11003)

[17] M. R. Murty AND S. Wong, The ABC conjecture and prime divisors of the Lucas and Lehmer sequences, Number Theory for the Millenium, III, (Urbana, IL, 2000), A. K. Peters, Natick, MA, 2002, 43-54. MR.1956267 (2003k:11058)

[18] Pace P. Nielsen, Odd perfect numbers have at least nine distinct prime factors, Math. Comp., to appear.

[19] O. ORE, On the averages of the divisors of a number, Amer. Math. Monthly, 55 (1948), 615-619. MR0027292 (10:284a)

[20] H. N. Shapiro, Introduction to the Theory of Numbers, Wiley, New York, 1983. MR693458 (84f:10001)

Department of Mathematics, Faculty of Science and Technology, Tokyo University of ScIEnce, NodA, Chiba, 278-8510, Japan

E-mail address: goto_takeshi@ma.noda.tus.ac.jp

Department of Mathematics, Kinki University Higashi-Osaka, Osaka 577-8502, Japan

E-mail address: ohno@math.kindai.ac.jp 